\title{
Top Quark Phenomenology: an overview
}

\author{
Rikkert Frederix \\ Institute for Theoretical Physics, University of Zurich, Winterthurerstrasse 190, 8057 Zurich, \\ Switzerland
}

DOI: http://dx.doi.org/10.5689/UA-PROC-2010-09/04

\begin{abstract}
In this talk three 2-sigma deviations from the Standard Model predictions in the top quark sector are briefly discussed. These are the excess of events in the tail of the $H_{T}$ distribution in $t \bar{t}$ events, the top-quark charge asymmetry and the discrimination of s- and t-channel events in single top. The latter has only been observed by CDF, while the other two are found by both CDF and D $\varnothing$.
\end{abstract}

\section{Top quarks at the Tevatron}

Everything we know about the top quark is coming from the experiments at the Tevatron collider at Fermilab. Since its discovery in 1995 [1], many of its properties have been firmly established over the years. The most precise measurements are its mass [2] and cross section [3], but results for other observables and properties of the top quark are improving as well. This includes: the top pair invariant mass distribution [4], the forward-backward charge asymmetry [5], the top quark width and its lifetime [6], the branching fraction to $W b$ compared to $W q$ [7], the $W$-boson helicity fractions and spin correlation in the top quark pair production [8], the top pair production mechanism ( $g g$ versus $q \bar{q}$ initial state) [9], the top quark's electric charge [10], the mass difference between top and anti-top quarks [11], the total single top cross section [12] including bounds on the CKM matrix element $\left|V_{t b}\right|$ [12] and discrimination between the singletop s- and t-channel production mechanisms [13]. Furthermore there have been many searches for new physics in the top quark sector, such as: resonant contributions to the $t \bar{t}$ production [4], search for fourth generation $t^{\prime}$ and $b^{\prime}$ quarks [14], scalar top admixture in $t \bar{t}$ events [15], top decays to charged Higgs bosons [16], $W^{\prime}$ and $H^{+}$resonant contributions to s-channel single top [17], anomalous $W t b$ couplings [18], production via a FCNC $(u(c)+g \rightarrow t)$ [19] and decay in $t \rightarrow Z q[20]$.

All measurements are in agreement with the Standard Model expectations within uncertainties. There are, however, three measurements that are slightly off: in the search for $t^{\prime}$ quarks, the tail of the $H_{T}$ distribution has a small excess of events, the top quark charge asymmetry is more pronounced than expected and the discrimination of the s- and t-channel cross section in single top is off by more than 2 sigma (CDF only). In the rest of this talk I'll give my personal view on these three experimental results. 


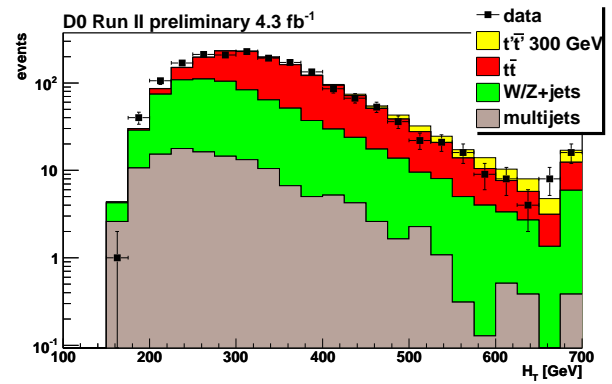

(a)

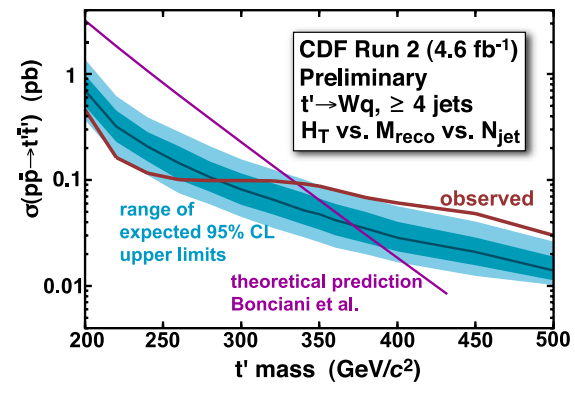

(b)

Figure 1: The $H_{T}$ distribution as measured by the $\mathrm{D} \varnothing$ collaboration (a). Observed (red curve) and expected (blue band) limits on the $t^{\prime}$ pair production cross section as a function of the $t^{\prime}$ mass as extracted from the data by CDF are shown (b). Figures are taken from Refs. [14].

\section{The three 2-sigma deviations}

\subsection{The tail of the $H_{T}$ distribution}

The $H_{T}$ observable is defined as the scalar sum of all transverse energies of the jets, leptons and missing $E_{T}$ and is a measure for the overall scale of the process. Recently both CDF and $\mathrm{D} \varnothing$ have updated their analysis for the search of heavy fourth generation quarks, in particular in $t^{\prime}$, i.e. a fourth generation top quark, that decays predominantly to $W^{+} b$. After selection cuts, the $H_{T}$ distribution shows an excess of events in the tail of the distribution. In fig. 1(a), this distribution is shown for $\mathrm{D} \varnothing$. As can be clearly seen from the last two bins (where the final bin is an overflow bin and contains also all events with $H_{T}>700 \mathrm{GeV}$ ) there are slightly more events than could have been expected from $t \bar{t}, W / Z+$ jets and multi-jet backgrounds. The inclusion of a heavy $t^{\prime}$ quark to the physics model, increases the number of events in the tail of the distribution and results in a better description of the data. This can also be seen from the plot in fig 1(b), where for a $t^{\prime}$ mass larger than about $350 \mathrm{GeV}$ the observed limit is at the border of the $95 \%$ confidence level for the predicted limit. This means that limit on the cross section for $t^{\prime}$ pairs is much milder than expected, which reflects the slight excess of events found in the tail of the $H_{T}$ distribution.

From a theoretical point of view, the tail of the $H_{T}$ distribution is difficult to model, because it is an observable quite sensitive to higher order corrections, see e.g. Ref. [21]. Furthermore, the approximation of describing extra radiation by a logarithm, as is done in all parton showers, does not capture hard radiation correctly, see e.g. Ref. [22]. Using a CKKW [23] or MLM [24] technique to merge higher multiplicity matrix elements with the parton shower could help in describing the process. This has been done for the $W+$ jets background, but not for the $t \bar{t}$ final state and therefore this might have an impact on the predictions and increase the number $t \bar{t}$ events in the tail and explain the slight excess.

Given that the effect is small (2 sigma) and non-trivial to model by Monte Carlo, it is not (yet) significant. More data, and in particular the LHC with its larger center-of-mass energy, 
and more refined analyses using matrix-elements with parton-shower matching will shed light on these effects.

\subsection{Top quark charge asymmetry}

In pure QCD production of top quark pairs at hadron colliders, the lowest order prediction shows no preferred direction for the top and anti-top quarks. When the next-to-leading corrections are taken into account, the interference between gluon radiation from the initial state quark line and the top quark line in the $q \bar{q} \rightarrow t \bar{t} g$ channel has a negative contribution to the charge asymmetry, while the contribution from the interference between the virtual box diagrams and the Born is positive. Furthermore there is a negligible contribution to the charge asymmetry from the flavor excitation processes $q g \rightarrow t \bar{t} q$. Top quark production by gluon fusion is symmetric in its initial state and therefore does not contribute to the asymmetry.

Quantitatively, the contribution from the virtual corrections is larger, hence the top quarks prefer to go in the direction of the incoming quark, and the anti-top quarks in the directions of the incoming anti-quark. Including the (LO) electroweak effects for the Tevatron the charge asymmetry is given by [25]

$$
\begin{aligned}
A_{F B}(\mathrm{lab}) & =0.051 \pm 0.006, \\
A_{F B}(t \bar{t}) & =0.078 \pm 0.009
\end{aligned}
$$

where the uncertainties are coming from renormalization and factorization scale dependence and

$$
\begin{aligned}
A_{F B}(\mathrm{lab}) & =\frac{\int_{y>0} N_{t}(y)-\int_{y>0} N_{\bar{t}}(y)}{\int_{y>0} N_{t}(y)+\int_{y>0} N_{\bar{t}}(y)} \\
A_{F B}(t \bar{t}) & =\frac{\int N(\Delta y>0)-\int N(\Delta y<0)}{\int N(\Delta y>0)+\int N(\Delta y<0)},
\end{aligned}
$$

are the definition of the charge asymmetry in the lab and the $t \bar{t}$ rest frames, respectively. Here, $N_{t(\bar{t})}(y)$ is the number of top (anti-top) quarks as a function of the rapidity $y$ and $\Delta y=y_{t}-y_{\bar{t}}$ the difference in rapidities between the top and anti-top quarks.

Recently, the prediction for the asymmetry in the top pair rest frame has been improved by including threshold logarithms at all orders and it was found that the results are quite stable under inclusion of these all-order effects [26],

$$
A_{F B}(t \bar{t})=0.073_{-0.007}^{+0.009}
$$

Both the CDF and $\mathrm{D} \varnothing$ collaborations measure a non-zero top quark charge asymmetry. CDF finds the following values [5]

$$
\begin{aligned}
& A_{F B}(\mathrm{lab})=0.073 \pm 0.028 \quad \mathrm{CDF} \\
& A_{F B}(t \bar{t})=0.057 \pm 0.028 \quad \mathrm{CDF} \text {, }
\end{aligned}
$$

while $\mathrm{D} \varnothing$ has only performed the analysis for the asymmetry in the top pair rest frame [5]

$$
A_{F B}(t \bar{t})=0.08 \pm 0.04 \text { (stat.) } \pm 0.01 \text { (syst.) } \quad \mathrm{D} \varnothing .
$$


However, these results are uncorrected for hadronization, underlying event, background effects, etc. which makes a direct comparsion with the predictions, eq. (1) and (3), unfair. The CDF collaboration provides also results corrected for these effects [5], which enhances the the asymmetry,

$$
\begin{array}{rll}
A_{F B}(\text { lab }) & =0.150 \pm 0.050 \text { (stat.) } \pm 0.024 \text { (syst.) } & \text { CDF (corrected) } \\
A_{F B}(t \bar{t}) & =0.158 \pm 0.072 \text { (stat. }) \pm 0.017 \text { (syst.) } & \text { CDF (corrected) }
\end{array}
$$

These measurements are almost two standard deviations larger than the theory predictions given in eq. (1) and (3). Notice that the uncorrected results from CDF and DO are in agreement with each other, and if we assume that the unfolding to parton level is the same for $\mathrm{D} \varnothing$ as it is for CDF, also the asymmetry as measured by $\mathrm{D} \varnothing$ is also about 2 sigma from its predicted value in perturbative QCD.

Even though the effect is around two standard deviations, and could therefore very well be a statistical effect, from the theory point of view many models beyond the SM were studied to see if these effects could be explained by new physics, see e.g. Ref. [27] and references therein. However, before assigning the large measured asymmetry to BSM effects, another option should be considered as well. Even though the NLO (i.e. first non-zero) predictions are stable under all-order resummation of threshold logarithms, this does not take into account that there might be a sizable effect from higher order virtual corrections, i.e. the two loop corrections to $q \bar{q} \rightarrow t \bar{t}$. The need to go from NLO to the full NNLO is acknowledged.

The ingredients to go one full order higher in perturbation theory are almost all known in analytic form [28] and the remaining contributions can be computed numerically [29]. The one important missing ingredient is the prescription to combine the contributions in an infra-red finite way. Several methods have been proposed [30], but non of them have been proven to work for top pair production in hadron collisions so far. Obtaining these results needs further theoretical developments.

From the experimental point of view the measurements are still statistically dominated, so with more data the measurements will become more precise. Due to the fact that the LHC as a proton-proton collider and that the initial state is dominated by gluons, measuring the asymmetry at this collider will be channeling at the least.

\subsection{Discrimination between s- and t-channel events by CDF}

Single top quark production has been firmly established with 5 standard deviations last year [31]. At the Tevatron only two of the three single top production channels contribute significantly, i.e. the s channel (with a cross section around $1 \mathrm{pb}$ ) and the t channel (about $2 \mathrm{pb}$ ). The total single top rate measured at the Tevatron agrees very well with the sum of the s- and t-channel cross section predictions.

For $\mathrm{D} \varnothing$ also the discrimination between the s- and t-channel cross section agrees well within 1 sigma with the theoretical predictions, see fig. 2(a). For CDF the situation is worse: the theoretical predictions are just outside the $95.5 \%$ C.L. contour, as can be seen in fig. 2(b). This discrimination is made using multi-variate techniques that use as much information as possible from the event topologies [13].

It is known that the t-channel process is difficult to model by Monte Carlo event generators due to the initial state $b$ quark. After showering of the events, the initial state b quark is modeled (in most cases) to come from an gluon splitting into a $b \bar{b}$ pair, for which the $\bar{b}$ quark 


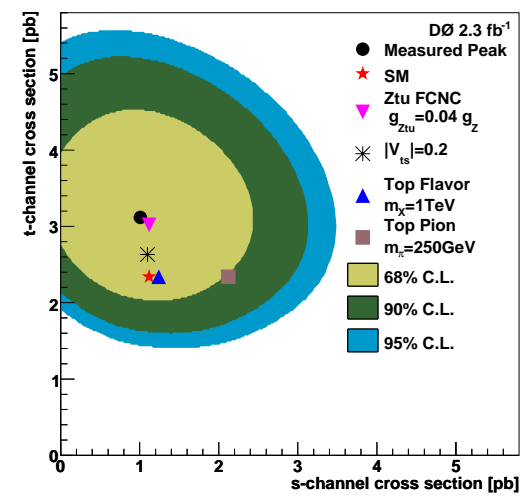

(a)

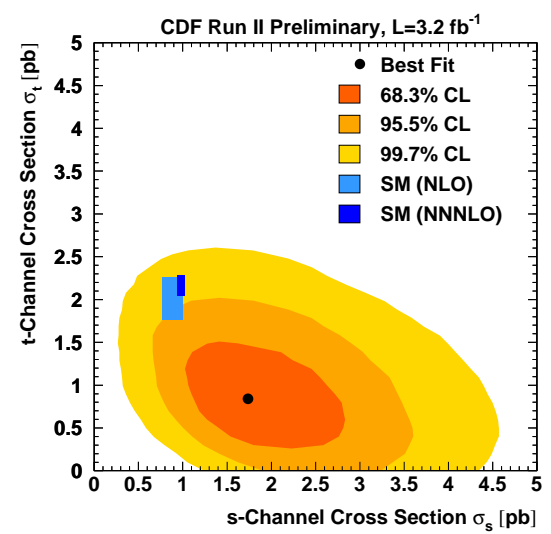

(b)

Figure 2: Posterior probability for s- and t-channel single top production by the $\mathrm{D} \varnothing$ (a) and CDF (b) collaborations. Figures are taken from Refs. [13].

becomes a final state parton, which is called the spectator- $b$ in the following. When only using the $2 \rightarrow 2$ process, the transverse momentum of the spectator- $b$ is greatly underestimated by the parton shower. This has been acknowledged and addressed by applying a merging procedure for leading order $2 \rightarrow 2$ and $2 \rightarrow 3$ events [32]. There is much freedom in the precise choice of the merging which can lead to a large spread in predictions for the spectator- $b$.

Recently, the NLO corrections to t-channel single top production in the 4-flavor scheme, i.e. starting from the $2 \rightarrow 3$ process, were performed [33]. This calculation gives the distributions for the spectator- $b$ quark for the first time at NLO, and should therefore be considered the predictions to validate the merging procedure of the $2 \rightarrow 2$ and $2 \rightarrow 3 \mathrm{MC}$ samples against.

Comparing the new predictions for the fraction of spectator- $b$ quarks with large transverse momentum $\left(p_{T}>20 \mathrm{GeV}\right)$ and small pseudo rapidities $(|\eta|<2)$ it has been found that results from the matching procedure as used by the D $\varnothing$ collaboration follows the NLO predictions quite well [34]. Unfortunately, for the Monte Carlo samples used by the CDF collaboration, which are based on the ZTOP program [35], this ratio is about half the size as the NLO prediction [36]. This means that CDF assumed that the t-channel events have only about half as many spectator- $b$ quarks as predicted by NLO. Given that s-channel events have an extra $b$ quark compared to the $2 \rightarrow 2$ t-channel events, at first sight this discrepancy could explain the measured excess of s-channel events compared to t-channel events: many t-channel events with a spectator- $b$ quark in the data were assigned to belong to the s-channel cross section. However, a careful improvement in the analysis by the CDF collaboration taking into account the new NLO computation, shows that this effect on the discrimination of the s- and t-channel cross sections is negligibly small [36]. Therefore, even with the improved theoretical predictions the 2 standard deviations discrepancy remains.

If the 2-sigma effect persists or increases when more data is collected and analysed by CDF, 
experimental effort to re-address the estimation of the uncertainties might be acknowledged. Also more theoretical work is needed to further improve predictions, e.g. by matching the NLO t-channel single top calculation in the 4-flavor scheme to a parton shower program following e.g. the MC@NLO [37] or POWHEG [38] methods.

\section{Conclusions}

There are three measurements in the top quark sector that are about 2 standard deviations away from its predictions within the Standard Model:

- There is a small excess of events in the tail of the $H_{T}$ distribution. Possible explanations might be that for the $H_{T}$ observable the tail of the distribution is known to be non-trivial to model using Monte Carlo event generators. In particular, the uncertainty from higher order corrections can be large. However, if the effect is truly there, a possible explanation is a fourth generation of quarks.

- The top quark charge asymmetry is found to be more pronounced than predicted by NLO QCD, which is the first order to give a non-zero result. The question arises if higher order effects might be important here, and it has been shown by using resummation techniques that this is probably not the case for the (soft) real emission. However, given that at NLO the virtual corrections give a larger contribution than the real emission, also the virtual corrections should be calculated at higher order to provide a prediction at high enough precision.

- The discrimination of s- and t-channel events in single top production by CDF. Even though in the Monte Carlo predictions used in the CDF analyses there were half as many spectator- $b$ quarks as compared to the recent NLO calculation, it does not explain the difference with $\mathrm{D} \varnothing$ nor with the theoretical predictions. There is no understanding yet of this peculiarity.

On the other hand, these three 2-sigma deviations could very well be statistical fluctuations. It is therefore important to see what this will give with more data, and what the LHC will tell is in the near future.

\section{References}

[1] CDF Collaboration, F. Abe et al., Phys. Rev. Lett. 74, 2626 (1995). DØ Collaboration, S. Abachi et al., Phys. Rev. Lett. 74, 2632 (1995).

[2] CDF \& DØ Collaboration, arXiv:1007.3178 [hep-ex].

[3] CDF Collaboration, Conf. Note 9913 (2009). DØ Collaboration, Conf. Note 6038 (2010).

[4] CDF Collaboration, T. Aaltonen et al., arXiv:0903.2850 [hep-ex]. DØ Collaboration, Conf. Note 5882 (2009).

[5] CDF Collaboration, Conf. Note 10185 (2010). DØ Collaboration, Conf. Note 6062 (2010).

[6] CDF Collaboration, Conf. Note 10035 (2010). DØ Collaboration, Conf. Note 6034 (2010).

[7] CDF Collaboration, D. E. Acosta et al., Phys. Rev. Lett. 95, 102002 (2005). DØ Collaboration, V. M. Abazov et al., Phys. Rev. Lett. 100, 192003 (2008).

[8] CDF Collaboration, Conf. Note 10211 (2010). DØ Collaboration, Conf. Note 5722 (2008). DØ Collaboration, Conf. Note 5950 (2009). 
[9] CDF Collaboration, T. Aaltonen et al., Phys.Rev. D78, 111101 (2008).

[10] CDF Collaboration, Conf. Note 9939 (2010). DØ Collaboration, V. Abazov et al., Phys.Rev.Lett. 98, 041801 (2007).

[11] CDF Collaboration, Conf. Note 10173 (2010). DØ Collaboration, V. Abazov et al., Phys.Rev.Lett. 103, 132001 (2009).

[12] CDF \& DØ Collaboration, T. E. W. Group, arXiv:0908.2171 [hep-ex].

[13] CDF Collaboration, T. Aaltonen et al., arXiv:1004.1181 [hep-ex]. D $\varnothing$ Collaboration, V. M. Abazov et al., Phys.Lett. B682, 363 (2010).

[14] CDF Collaboration, Conf. Note 10110 (2010). DØ Collaboration, Conf. Note 5892 (2010).

[15] DØ Collaboration, V. Abazov et al., Phys.Lett. B674, 4 (2009).

[16] CDF Collaboration, Conf. Note 10104 (2010). DØ Collaboration, V. Abazov et al., Phys.Lett. B682, 278 (2009).

[17] CDF Collaboration, T. Aaltonen et al., Phys.Rev.Lett. 103, 041801 (2009). DØ Collaboration, V. Abazov et al., Phys.Rev.Lett. 100, 211803 (2008). DØ Collaboration, V. Abazov et al., Phys.Rev.Lett. 102, 191802 (2009).

[18] DØ Collaboration, V. Abazov et al., Phys.Rev.Lett. 102, 092002 (2009).

[19] CDF Collaboration, T. Aaltonen et al., Phys.Rev.Lett. 102, 151801 (2009). DØ Collaboration, V. M. Abazov et al., arXiv:1006.3575 [hep-ex].

[20] CDF Collaboration, T. Aaltonen et al., Phys.Rev.Lett. 101, 192002 (2008).

[21] M. Rubin, G. P. Salam and S. Sapeta, arXiv:1006.2144 [hep-ph].

[22] J. Alwall, S. de Visscher and F. Maltoni, JHEP 0902, 017 (2009).

[23] S. Catani, F. Krauss, R. Kuhn and B. Webber, JHEP 0111, 063 (2001).

[24] J. Alwall et al., Eur.Phys.J. C53, 473 (2008).

[25] J. H. Kuhn and G. Rodrigo, Phys.Rev.Lett. 81, 49 (1998). J. H. Kuhn and G. Rodrigo, Phys.Rev. D59, 054017 (1999).

[26] L. G. Almeida, G. F. Sterman and W. Vogelsang, Phys.Rev. D78, 014008 (2008). V. Ahrens, A. Ferroglia, M. Neubert, B. D. Pecjak and L. L. Yang, arXiv:1003.5827 [hep-ph].

[27] Q.-H. Cao, D. McKeen, J. L. Rosner, G. Shaughnessy and C. E. M. Wagner, Phys. Rev. D81, 114004 (2010).

[28] R. Bonciani, A. Ferroglia, T. Gehrmann, D. Maitre and C. Studerus, JHEP 0807, 129 (2008). R. Bonciani, A. Ferroglia, T. Gehrmann and C. Studerus, JHEP 0908, 067 (2009). J. G. Korner, Z. Merebashvili and M. Rogal, Phys. Rev. D77, 094011 (2008). S. Dittmaier, P. Uwer and S. Weinzierl, Phys. Rev. Lett. 98, 262002 (2007).

[29] M. Czakon, Phys.Lett. B664, 307 (2008).

[30] A. Gehrmann-De Ridder, T. Gehrmann and E. W. N. Glover, JHEP 09, 056 (2005). S. Catani and M. Grazzini, Phys. Rev. Lett. 98, 222002 (2007). M. Czakon, Phys.Lett. B693, 259 (2010). G. Somogyi, Z. Trocsanyi and V. Del Duca, JHEP 0506, 024 (2005).

[31] DØ Collaboration, . V. M. Abazov, arXiv:0903.0850 [hep-ex]. CDF Collaboration, T. Aaltonen et al., arXiv:0903.0885 [hep-ex].

[32] E. E. Boos, V. E. Bunichev, L. V. Dudko, V. I. Savrin and A. V. Sherstnev, Phys. Atom. Nucl. 69, 1317 (2006).

[33] J. M. Campbell, R. Frederix, F. Maltoni and F. Tramontano, JHEP 10, 042 (2009). J. M. Campbell, R. Frederix, F. Maltoni and F. Tramontano, Phys. Rev. Lett. 102, 182003 (2009).

[34] SM and NLO Multileg Working Group Collaboration, J. R. Andersen et al., arXiv:1003.1241 [hep-ph].

[35] Z. Sullivan, Phys. Rev. D70, 114012 (2004).

[36] CDF Collaboration, J. Lueck, Private Communication .

[37] S. Frixione and B. R. Webber, JHEP 06, 029 (2002).

[38] P. Nason, JHEP 11, 040 (2004). 